\title{
Non-invasive capnodynamic mixed venous oxygen saturation during major changes in oxygen delivery
}

\author{
Anders Svedmyr ${ }^{1,2}$ (D) Mark Konrad ${ }^{3} \cdot$ Mats Wallin $^{1,3} \cdot$ Magnus Hallbäck $^{3}$ (D) Per-Arne Lönnqvist ${ }^{1,2}$ (D) \\ Jacob Karlsson ${ }^{1,2}$ (D)
}

Received: 16 July 2021 / Accepted: 27 September 2021 / Published online: 5 October 2021

(c) The Author(s) 2021

\begin{abstract}
Mixed venous oxygen saturation $\left(\mathrm{SvO}_{2}\right)$ is an important variable in anesthesia and intensive care but currently requires pulmonary artery catheterization. Recently, non-invasive determination of $\mathrm{SvO}_{2}\left(\mathrm{Capno}-\mathrm{SvO}_{2}\right)$ using capnodynamics has shown good agreement against CO-oximetry in an animal model of modest hemodynamic changes. The purpose of the current study was to validate Capno- $\mathrm{SvO}_{2}$ against CO-oximetry during major alterations in oxygen delivery. Furthermore, evaluating fiberoptic $\mathrm{SvO}_{2}$ for its response to the same challenges. Eleven mechanically ventilated pigs were exposed to oxygen delivery changes: increased inhaled oxygen concentration, hemorrhage, crystalloid and blood transfusion, preload reduction and dobutamine infusion. Capno-SvO $\mathrm{S}_{2}$ and fiberoptic $\mathrm{SvO}_{2}$ recordings were made in parallel with CO-oximetry. Respiratory quotient, needed for capnodynamic $\mathrm{SvO}_{2}$, was measured by analysis of mixed expired gases. Agreement of absolute values between CO-oximetry and Capno- $\mathrm{SvO}_{2}$ and fiberoptic $\mathrm{SvO}_{2}$ respectively, was assessed using Bland-Altman plots. Ability of Capno- $\mathrm{SvO}_{2}$ and fiberoptic $\mathrm{SvO}_{2}$ to detect change compared to CO-oximetry was assessed using concordance analysis. The interventions caused significant hemodynamic variations. Bias between Capno-SvO $\mathrm{S}_{2}$ and $\mathrm{CO}$-oximetry was $+3 \%$ points $\left(95 \%\right.$ limits of agreements -7 to +13 ). Bias between fiberoptic $\mathrm{SvO}_{2}$ and CO-oximetry was $+1 \%$ point, (95\% limits of agreements -7 to +9 ). Concordance rate for $\mathrm{Capno}_{-} \mathrm{SvO}_{2}$ and fiberoptic $\mathrm{SvO}_{2}$ vs. CO-oximetry was $98 \%$ and $93 \%$, respectively. Capno- $\mathrm{SvO}_{2}$ generates absolute values close to $\mathrm{CO}$-oximetry. The performance of Capno-SvO $\mathrm{V}_{2}$ vs. COoximetry was comparable to the performance of fiberoptic $\mathrm{SvO}_{2}$ vs. CO-oximetry. Capno-SvO $\mathrm{O}_{2}$ appears to be a promising tool for non-invasive $\mathrm{SvO}_{2}$ monitoring.
\end{abstract}

Keywords Hemodynamic monitoring $\cdot$ Carbon dioxide $\cdot$ Cardiac output $\cdot$ Pulmonary artery $\cdot$ Oxygen $\cdot$ Oximetry

\section{Introduction}

Mixed venous oxygen saturation $\left(\mathrm{SvO}_{2}\right)$ is a clinically useful monitoring parameter that provide important information regarding the whole-body oxygen delivery/consumption

Jacob Karlsson

jacob.karlsson.1@ki.se

1 Karolinska Institute Department of Physiology and Pharmacology (FYFA), C3, Per-Arne Lönnqvist Group Section of Anesthesiology and Intensive Care, Anestesi- och Intensivvårdsavdelningen, 17176 Stockholm, PA, Sweden

2 Pediatric Perioperative Medicine and Intensive Care, Karolinska University Hospital, Eugenivägen 23, 17164 Stockholm, Sweden

3 Maquet Critical Care AB, Röntgenvägen 2, 17106 Solna, Sweden balance and is of particular interest in the setting of anaesthesia and intensive care [1]. The use of $\mathrm{SvO}_{2}$ as a routine monitoring parameter has so far been hampered by the fact that intermittent blood sampling from the pulmonary artery (CO-oximetry; representing "gold standard") as well as continuous assessment by fibreoptic spectrophotometry both require invasive catherization of the pulmonary artery, which is associated with certain risks as well as increased nursing care demands and cost $[2,3]$.

During our development of a modern version of capnodynamic assessment of cardiac output (Effective Pulmonary Blood Flow, $\mathrm{CO}_{\mathrm{EPBF}}$ ), which we have found to produce similar results when compared to accepted reference methods, we have come to realize that combining $\mathrm{CO}_{\mathrm{EPBF}}$ with the respiratory quotient (RQ) results in the possibility to determine $\mathrm{SvO}_{2}$ noninvasively [4-7]. In a recent proof-ofconcept study we have found that this new non-invasive and 
continuous methodology of monitoring $\mathrm{SvO}_{2}\left(\mathrm{Capno}-\mathrm{SvO}_{2}\right)$ show promising results when compared to $\mathrm{CO}$-oximetry determinations of $\mathrm{SvO}_{2}$ in a porcine model, during modest hemodynamic challenges [8].

The aim of the present study was to validate Capno$\mathrm{SvO}_{2}$ against the gold standard CO-oximetry for agreement of absolute values and ability to detect change during profound variations in oxygen delivery while measuring RQ, using our previously well-established porcine model. A comparison between CO-oximetry and another continuous $\mathrm{SvO}_{2}$ methodology, i.e., fibreoptic $\mathrm{SvO}_{2}$, was also included for comparison.

\section{Materials and methods}

\subsection{Animal preparations}

The study was performed at the Hedenstierna Laboratory, Uppsala University, Uppsala Sweden. Authorization was granted from Uppsala Animal Ethics Committee (Uppsala, Sweden case number C75/16, chairperson Erik Göransson on August 26, 2016).

The animals were handled in accordance with the animal experimentation guidelines of the Uppsala Animal Ethics Committee and Animal Research: Reporting of In Vivo Experiments (ARRIVE) guidelines.

Eleven domestic-breed pigs of both sexes (median weight $29.6 \mathrm{~kg}$, range $27.1-31.7 \mathrm{~kg}, 6-8$ weeks of age), from the same breeding colony (Mångsbo Farm, Uppsala, Sweden) were used. The animals were kept in a light- and temperature-controlled environment, with unlimited access to tap water and food on a standardized schedule. The experiments were done during daytime hours.

The pigs were anaesthetized as previously described and mechanically ventilated in a volume-controlled mode (Servo-I; Maquet, Solna, Sweden), with tidal volume of $10 \mathrm{~mL} \mathrm{~kg}^{-1}$ and fraction of inspired oxygen $\left(\mathrm{FiO}_{2}\right) 0.3$ [9]. Positive end-expiratory pressure (PEEP) was kept at $5 \mathrm{~cm}$ $\mathrm{H}_{2} \mathrm{O}$ after an initial 2-min period of lung expansion using PEEP $10 \mathrm{~cm} \mathrm{H}_{2} \mathrm{O}$ as previously described [8]. After the lung expansion manoeuvre, an air test using $\mathrm{FiO}_{2} 0.21$ was performed and repeated if necessary, aiming for sustained pulse oximetry saturation $>97 \%$ as indicative of open lung conditions [10]. The animals were given a bolus of Ringers' acetate solution $20 \mathrm{~mL} \mathrm{~kg}^{-1}$ after induction and thereafter kept on maintenance infusion of glucose $25 \mathrm{mg} \mathrm{mL}^{-1} 8$ $\mathrm{mL} \mathrm{kg}{ }^{-1} \mathrm{~h}^{-1}$ and Ringer's acetate solution $10 \mathrm{~mL} \mathrm{~kg}^{-1} \mathrm{~h}^{-1}$. Exhaled $\mathrm{CO}_{2}$ was measured by a mainstream infrared $\mathrm{CO}_{2}$ sensor (Capnostat-3; Respironics Inc, Wallingford, CT), and ventilation airflow was registered through the regular flow sensor of the servo-I ventilator. Volumetric capnography was utilised for calculating the $\mathrm{CO}_{2}$ elimination rate $\left(\mathrm{VCO}_{2}\right)$, as described in previous studies [11].

Adequate anesthetic depth and analgesic level were tested regularly during the experiment according to standard procedures of the laboratory. The animals were fitted with monitoring devices described in detail in previous studies [9]. In addition a $7.5 \mathrm{~F}$ pulmonary artery catheter with fibreoptic $\mathrm{SvO}_{2}$ monitoring (Swan-Gantz pulmonary artery catheter, model 774F75; Edwards Lifesciences, Irvine CA; USA) and a $5 \mathrm{~F}$ femoral artery cannula for transpulmonary thermodilution cardiac output monitoring (PICCO2 ${ }^{\mathrm{TM}}$, Pulsion Medical Systems, Munich, Germany), were also included as well as an additional $7.5 \mathrm{~F}$ pulmonary artery introducer sheath for rapid fluid administration.

A $12 \mathrm{~F}$ balloon tipped catheter (Mediq Sverige AB, Uppsala, Sweden) was surgically introduced through the left femoral vein, to a depth of $30 \mathrm{~cm}$ corresponding to the inferior vena cava (IVC). The catheter was fitted with a $10 \mathrm{~mL}$ balloon allowing partial IVC occlusion when inflated with $\mathrm{NaCl} 0.9 \%$. The animals were given a bolus dose of intravenous heparin $5000 \mathrm{U}$ (LEO pharma) to minimize the risk of clotting due to the extensive intravascular monitoring setup.

$\mathrm{SvO}_{2}$ through blood samples were analysed by a COoximeter calibrated for porcine hemoglobin (OSM3; Radiometer Medical AbS, Brønshøj, Denmark). This also provided the hemoglobin level, necessary for the calculations of $\mathrm{CO}_{\mathrm{EPBF}}$ and Capno- $\mathrm{SvO}_{2}$ as well as for calibrations of fiberoptic $\mathrm{SvO}_{2}$.

\subsection{Assessment of Capno-SvO}

The principle behind continuous Capno- $\mathrm{SvO}_{2}$ method has been described in detail in previous work by our research team [8]. The Capno- $\mathrm{SvO}_{2}$ method is based on the differential Fick principle and utilizes a combination of continuous estimation of $\mathrm{CO}_{\mathrm{EPBF}}$ and oxygen consumption $\left(\mathrm{VO}_{2}\right)$ incorporated in a rearranged Fick's equation [12]. $\mathrm{CO}_{\mathrm{EPBF}}$ is estimated by applying a special breathing pattern of variations in I:E relationships (six breaths with normal I:E relations followed by three breaths vid an approximately $2 \mathrm{~s}$ expiratory pause). This breathing pattern causes small fluctuations in alveolar $\mathrm{CO}_{2}$ concentration and $\mathrm{VCO}_{2}$, related to the pulmonary blood flow participating in gas exchange which allows for estimation of $\mathrm{CO}_{\mathrm{EPBF}}[5,7,13] . \mathrm{VO}_{2}$ can be estimated using continuous volumetric capnography measurement of $\mathrm{VCO}_{2}$ combined with RQ:

$V O_{2}=\frac{V C O_{2}}{R Q}$

where $\mathrm{VO}_{2}$ is the oxygen consumption $\left(\mathrm{mL} \mathrm{min}^{-1}\right), \mathrm{VCO}_{2}$ is the $\mathrm{CO}_{2}$ production rate $\left(\mathrm{mL} \mathrm{min}^{-1}\right), \mathrm{RQ}$ is the respiratory quotient. 
$\mathrm{VCO}_{2}$ applied in Eq. 1 is determined as a moving mean value of measured $\mathrm{CO}_{2}$ elimination made over a period of 20 min in order to produce stable $\mathrm{VCO}_{2}$ values with the intent to reflect an assumed stable metabolism.

Using Fick's equation for oxygen, the content of oxygen in mixed venous blood $\left(\mathrm{CvO}_{2}\right)$ can be derived:

$C O_{E P B F}=\frac{V O_{2}}{\mathrm{CcO}_{2}-\mathrm{CvO}_{2}}$

where $\mathrm{CO}_{\mathrm{EPBF}}$ is the effective pulmonary blood flow ( $\mathrm{L}$ $\left.\min ^{-1}\right), \mathrm{VO}_{2}$ is the oxygen consumption $\left(\mathrm{mL} \mathrm{min}^{-1}\right), \mathrm{CcO}_{2}$ is the pulmonary end capillary oxygen content $\left(\mathrm{mL} \mathrm{L}^{-1}\right)$, $\mathrm{CvO}_{2}$ is the pulmonary mixed venous oxygen content $(\mathrm{mL}$ $\left.\mathrm{L}^{-1}\right)$.

Equation 2 can be further rearranged to derive $\mathrm{CvO}_{2}$ :

$\mathrm{CvO}_{2}=\mathrm{CcO}_{2}-\frac{V \mathrm{O}_{2}}{C \mathrm{O}_{E P B F}}$

where $\mathrm{CO}_{\mathrm{EPBF}}$ is the effective pulmonary blood flow ( $\mathrm{L}$ $\left.\min ^{-1}\right), \mathrm{VO}_{2}$ is the oxygen consumption $\left(\mathrm{mL} \mathrm{min}^{-1}\right), \mathrm{CcO}_{2}$ is the pulmonary end capillary oxygen content $\left(\mathrm{mL} \mathrm{L}^{-1}\right)$, $\mathrm{CvO}_{2}$ is the pulmonary mixed venous oxygen content $(\mathrm{mL}$ $\left.\mathrm{L}^{-1}\right)$.

$\mathrm{CvO}_{2}$ from Eq. 3 can then be used to calculated $\mathrm{SvO}_{2}$ by using the solubility constant for oxygen in blood plasma, Hüfner's constant and the hemoglobin value as previously described thereby generating breath-by-breath estimations of $\mathrm{SvO}_{2}$ [8].

\subsection{RQ-measurements}

The RQ was determined immediately before the start of the first challenge (morning) and immediately before the start of the last challenge (afternoon), approximately $4 \mathrm{~h}$ apart (shown in Fig. 1). Measurements were performed in all animals, by analysis of mixed expired gas collected in a Douglas bag. Each RQ measurement was conducted in the following way:

- Ventilator bias flow during expiration phase ((normally $2 \mathrm{~L} \mathrm{~min}^{-1}$ in Servo-I ventilator (Maquet, Solna, Sweden)) was turned off to avoid diluting the gas in the bag with inspiration gas.

- $\mathrm{FiO}_{2}$ was changed from 0.30 to 0.21 (i.e., room air), which was done for two reasons; it gives the best conditions for the RQ calculation (as shown in Eq. 1 below) and the inspired gas concentration is well-defined without relying on accurate gas mixing by the ventilator.

- To reach a stable state of nitrogen $\left(\mathrm{N}_{2}\right), 20$ min wash-in of $\mathrm{N}_{2}$ was allowed after the change of $\mathrm{FiO}_{2}$ and before the bag was connected to the ventilator exhaust port.

- Gas was collected for about $10 \mathrm{~min}$; roughly $70 \mathrm{~L}$ of expiratory gas was collected.

- The content of the bag was analyzed using the sidestream gas analyzer of a Flow-i anesthesia system (Maquet, Solna, Sweden). Gas was alternatingly sampled from the bag or from the room air.

Gas concentrations of $\mathrm{FiO}_{2}$ (room air), $\mathrm{FemixO}_{2}$ (bag) and $\mathrm{FemixCO}_{2}$ (bag) were measured at ATPD state (Ambient temperature and pressure, dry). RQ was calculated using the following formula, which is based on the Haldane transformation to account for the imbalance between inspiration and expiration volumes:

$R Q=\frac{\left(1-\mathrm{FiO}_{2}\right) \cdot \mathrm{Femix}_{2}}{\left(1-\mathrm{Femix}_{2} \mathrm{CO}_{2}\right) \cdot \mathrm{FiO}_{2}-\mathrm{FemixO}_{2}}$

$\mathrm{RQ}$ is the respiratory quotient, $\mathrm{FiO}_{2}$ is the fraction of inspired oxygen, $\mathrm{Femix}_{2} \mathrm{CO}_{2}$ is the carbon dioxide concentration in the Douglas bag, FemixO $\mathrm{O}_{2}$ is the oxygen concentration in the Douglas bag.

A detailed derivation of the RQ-equation can be found in Supplemental Description Content 1.

\subsection{Study protocol}

After preparation, the animals were allowed a 20-minute stabilizing period. Following this, an arterial blood sample was taken for calibration of the fiberoptic ( $\mathrm{Hb}$ and CO-oximetry $\mathrm{SvO}_{2}$ as per the manufacturers recommendation) and the capnodynamic monitoring system (Hb). Subsequently, five paired baseline measurement of all three methods were performed, $1 \mathrm{~min}$ apart, for calculation of

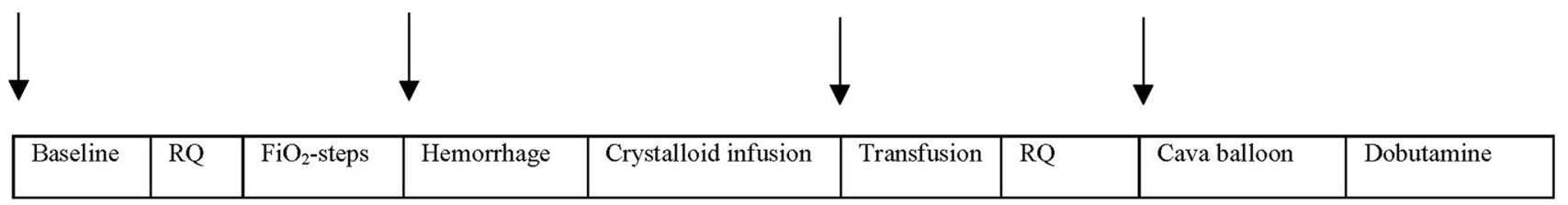

Fig. 1 Scheme of the study protocol. $R Q$ respiratory quotient; $\left(\mathrm{FiO}_{2}\right), \mathrm{FiO}_{2}$ steps. Black arrows indicate calibration points were fiberoptic $\mathrm{SvO} \mathrm{O}_{2}$ was calibrated against CO-oximetry and hemoglobin and Capno- $\mathrm{SvO}_{2}$ against current hemoglobin 
inherent precisions. After this, the animals were subjected to six different hemodynamic challenges anticipated to affect $\mathrm{SvO}_{2}$ (Fig. 1):

1. Stepwise increase in $\mathrm{FiO}_{2}:(0.21-0.4-0.75-1.0)$. Each $\mathrm{FiO}_{2}$ level was applied for $3 \mathrm{~min}$ and simultaneous $\mathrm{SvO}_{2}$ recordings were made at the end of each $\mathrm{FiO}_{2}$ level. After this, $\mathrm{FiO}_{2}$ was returned to baseline 0.3 and an air test was performed to ensure open lung conditions after $\mathrm{FiO}_{2} 1.0$ and lungs expanded as described if needed as described above.

2. Hemorrhage: $15 \mathrm{~mL} \mathrm{~kg}^{-1}$ of blood were drawn under 5-10 min and kept in a plastic bag intended for donor blood, containing $5000 \mathrm{U}$ of Heparin (LEO pharma). $\mathrm{SvO}_{2}$ values were recorded just before and $5 \mathrm{~min}$ after the hemorrhage.

3. Crystalloid bolus: $15 \mathrm{~mL} \mathrm{~kg}^{-1}$ of Ringer's acetate solution was given centrally in the 7.5 F PA introducer sheath to simulate crystalloid resuscitation of acute hypovolemia, over 5 min, 6 simultaneous $\mathrm{SvO}_{2}$ measurements were obtained: before crystalloid infusion and $0,5,10,15$ and 20 min after completions of infusion where 0 is just after completed infusion.

4. Blood transfusion: blood drawn under step 2 was transfused back over 5-10 min and $\mathrm{SvO}_{2}$ data were recorded before transfusion and $0,5,10,15$ and 20 min after completion of the transfusion where 0 is just after completed infusion.

5. Cava balloon: the cava balloon was inflated (reducing cardiac output by $30-50 \%$ ). $\mathrm{SvO}_{2}$ data was collected prior to inflating the balloon, 2 min after inflation, and 3 min after deflation.

6. Dobutamine: a central infusion of dobutamine (Hameln pharma gmbh, Hameln, Germany) $10 \mathrm{mcg} \mathrm{kg}^{-1} \mathrm{~min}^{-1}$ was started immediately after the 3-min recording after cava balloon deflation. $\mathrm{SvO}_{2}$ was then recorded 5, 10 and 15 min after starting the dobutamine infusion.

The fiberoptic $\mathrm{SvO}_{2}$ measurement was calibrated with $\mathrm{Hb}$-level and CO-oximetry $\mathrm{SvO}_{2}$ values as per manufacturer's instructions. This was performed totally 4 times during the experiment as marked in Fig. 1 to ensure continuous optimal performance during the experiment. At the same time points, the capnodynamic method was calibrated with the corresponding Hb-level. Throughout the study protocol, Capno-SvO $\mathrm{S}_{2}$ and fiberoptic $\mathrm{SvO}_{2}$, were recorded just before the blood samples for CO-oximetry were drawn. Due to previous experiences from caval occlusion causing extensive hemodynamic strain on the animals, no baseline recordings were made before the dobutamine step. Instead, dobutamine was started immediately after the last recording of step 5 as described above. The 3-minute recordings after cava balloon deflation, i.e., just before start of the dobutamine infusion, was used as comparison point for concordance analysis for the dobutamine step.

The animals were euthanized at the end of the experiment, according to the laboratory's routines.

\subsection{Statistical analysis}

Raw data for absolute values of all three $\mathrm{SvO}_{2}$ methods (CO-oximetry, Capno- $\mathrm{SvO}_{2}$ and fibreoptic $\mathrm{SvO}_{2}$ ), as well as paired differences between Capno-SvO ${ }_{2}$ and $\mathrm{CO}$-oximetry and between fibreoptic $\mathrm{SvO}_{2}$ and $\mathrm{CO}$-oximetry, were controlled for normal distribution by D'Agostino and Pearson test and visual inspection of the corresponding histograms. Values are presented as mean and $95 \%$ confidence interval (CI).

The first five measurements, during hemodynamically stable baseline conditions, were used for calculation of inherent precision for each $\mathrm{SvO}_{2}$ method respectively. Inherent precision was defined as 2 times the coefficient of variation $(\mathrm{CV}=\mathrm{SD} / \mathrm{mean})$ and was in turn used to quantify the least significant change (LSC) for the reference method CO-oximetry [14]. The LSC reflects the minimum change measured and recognized as a true change [14]. LSC was then used to determine the exclusion zone in the concordance analysis as previously described [8].

Bland-Altman analysis corrected for repeated measurements was used to assess agreement of absolute values between paired recording of $\mathrm{CO}$-oximetry and Capno$\mathrm{SvO}_{2}$ as well as between CO-oximetry and fibreoptic $\mathrm{SvO}_{2}$. Bias was defined as the mean difference between the tested methods and the reference method $[15,16]$. Limits of agreement (bias $\pm[1.96 \times \mathrm{SD}]$ ) were used when presenting the spread of included data points and are shown with the corresponding 95\% CI. Mean percentage error (PE) was calculated as $1.96 \times \mathrm{SD}$ of the differences, divided by the mean of the reference method [17]. A predefined PE of $<30 \%$ was defined a priori to indicate a clinically useful agreement. The predefined maximum allowed difference for agreement between the tested methods and the reference method was set to $15 \%$ points (based on previous published performance of fibreoptic $\mathrm{SvO}_{2}$ devices and Capno$\mathrm{SvO}_{2}$ ) [8, 18-20]. Based on our previous study, given the maximally allowed difference between the tested method and the reference method, a minimum of approximately 250 paired data points (corresponding to 10 animals with 25 paired recording per animal) was required to show that the 95\% confidence interval of the limits of agreement would fall within the pre-defined maximal allowed difference thus showing that the methods are in agreement (80\% power, 0.05 significance level) $[8,15,21]$. The ability of Capno- $\mathrm{SvO}_{2}$ and fibreoptic $\mathrm{SvO}_{2}$ to track changes in $\mathrm{SvO}_{2}$ compared to the refence method, was assessed by calculating the concordance rate, i.e., the percentage 
of data points moving in the same direction when comparing two different techniques [22]. Recorded changes between baseline measurements before each hemodynamic intervention, and the value corresponding to the highest change from baseline within that intervention, were used for calculation of the concordance rate. The LSC for COoximetry was found to be $9.5 \%$. Based on this, changes in $\mathrm{SVO}_{2}$ of less than $9.5 \%$ (rounded up to $10 \%$ ) of the mean of all $\mathrm{SvO}_{2} \mathrm{CO}$-oximetry recordings (corresponding to a minimal change in CO-oximetry $\mathrm{SvO}_{2}$ of $5 \%$ points) were not regarded as true changes and excluded from the concordance analysis [14].

Due to the increasingly unreliable performance of CO-oximetry method for low $\mathrm{SvO}_{2}$ levels, i.e., for values under 30\%, CO-oximetry $\mathrm{SvO}_{2}$ values under $30 \%$ and its associated Capno-SvO $\mathrm{Sv}_{2}$ and fibreoptic $\mathrm{SvO}_{2}$ values were excluded in the analysis [23, 24].

GraphPad Prism (version 9.0.0 for Windows, GraphPad Software, San Diego, CA, USA) and Medcalc Statistical Software version 16.8.4 (MedCalc Software, Ostend, Belgium) was used for statistical calculations and Microsoft Excel for Mac 2020 version 16.41 for data handling.

\section{Results}

All animals survived the experiment. $\mathrm{SvO}_{2}$ data for step 1-3 in the protocol was excluded from one animal due malposition of the PA-catheter which was then repositioned correctly for step $4-6$. The dobutamine step was introduced after the first animal and thus includes only 10 animals.

\subsection{Calculated inherent precision}

The inherent precision was found to be $\pm 7 \%$ for $\mathrm{CO}$-oximetry $\mathrm{SvO}_{2}$ and $\pm 8 \%$ and $\pm 6 \%$ for Capno-SvO $\mathrm{S}_{2}$ and fiberoptic $\mathrm{SvO}_{2}$, respectively which is in line with previous publications $[8,19]$.

\section{$3.2 \mathrm{RQ}$}

The mean RQ for morning and afternoon recordings for all eleven animals, i.e., in total $22 \mathrm{RQ}$ values, was found to be 0.97 (range 0.88-1.06) and RQ 0.97 was then used for all $\mathrm{SvO}_{2}$-capno calculations.

\subsection{Response to hemodynamic interventions}

All animals displayed $\mathrm{SvO}_{2}$ reactions in an anticipated manner when exposed to the various hemodynamic interventions. Both Capno- $\mathrm{SvO}_{2}$ and fiberoptic $\mathrm{SvO}_{2}$ showed parallel changes when compared to the reference method CO-oximetry with increase in $\mathrm{SvO}_{2}$ in response to increased $\mathrm{FiO}_{2}$, crystalloid infusion, blood transfusion and dobutamine administration. Correspondingly, hemorrhage and partial cava occlusion led to decreases in $\mathrm{SvO}_{2}$ for all methods. The changes in $\mathrm{SvO}_{2}$ for each hemodynamic intervention for all three $\mathrm{SvO}_{2}$ methods are shown in the event plot in Fig. 2.
Fig. 2 Event plot showing $\mathrm{SvO}_{2}$ responses to the various hemodynamic challenges for all three $\mathrm{SvO}_{2}$ monitoring methods. Values are mean $(95 \% \mathrm{CI}) . \mathrm{N}=11$. Arrows indicate recalibration points for $\mathrm{SvO}_{2}$-capno and fiberoptic $\mathrm{SvO}_{2}$. $B L$ Baseline, $B L_{\text {mean }}$ mean of precision recordings, $\mathrm{FiO}_{2}$ fraction inspired oxygen; $B L_{\text {cava }}$ Baseline cava occlusion step, Cavaoccl. 2 min 2 min after cava occlusion, Cava defl. 3 min 3 min after cava balloon deflation

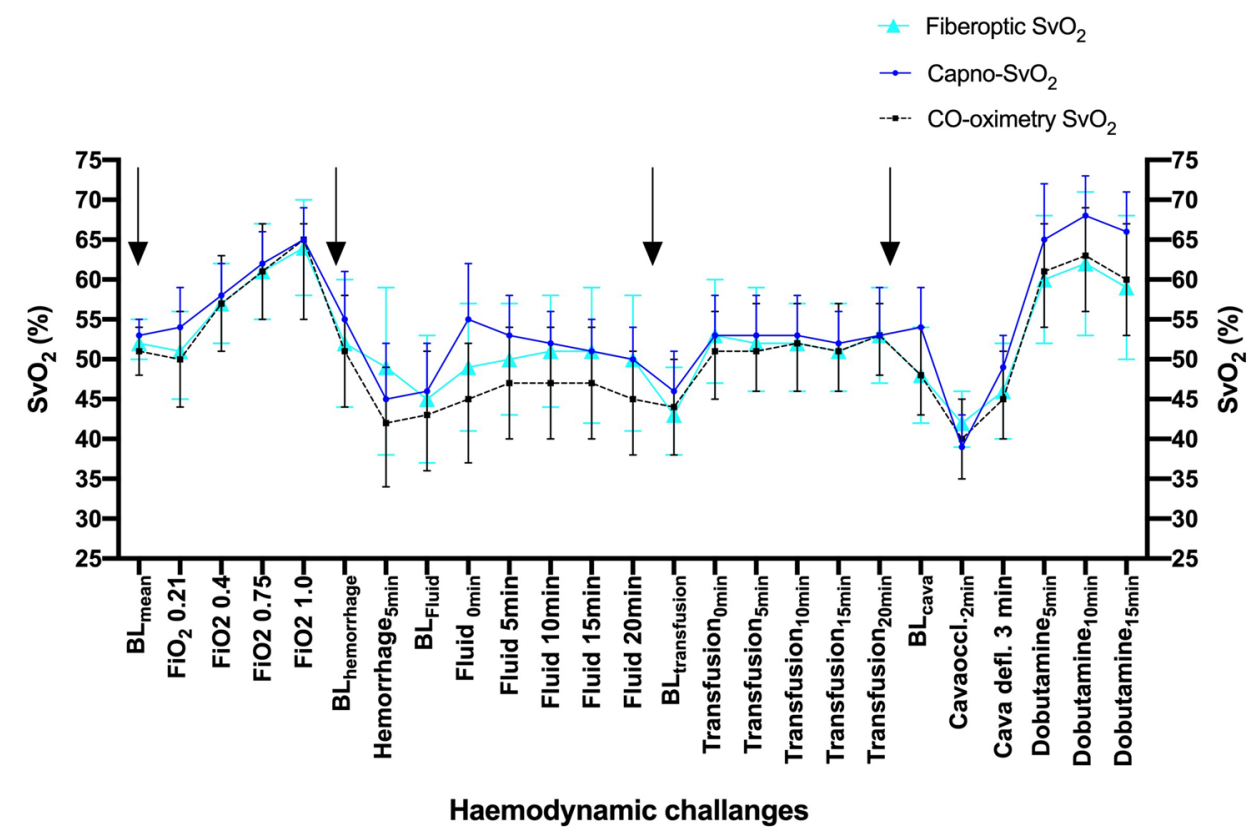




\subsection{Agreement of absolute values}

Bland-Altman analysis for all paired data showed a bias between Capno-SvO $\mathrm{Sv}_{2}$ and $\mathrm{CO}$-oximetry of $+3 \%$ points with $95 \%$ limits agreement of -7 (CI -10 to -4 ) to +13 (CI 11 to 17 ) percentage points and a mean percentage error of $18 \%$. Correspondingly, the bias between fiberoptic $\mathrm{SvO}_{2}$ and CO-oximetry was found to be $+1 \%$ point with $95 \%$ limits of agreement of -7 (CI -8 to -6$)$ to +9 (CI 8 to 11$)$ percentage points and PE 16\%. Bland Altman plots for Capno-SvO vs. CO-oximetry and fiberoptic $\mathrm{SvO}_{2}$ vs. CO-oximetry are shown in Fig. 3. Table 1 shows the agreement of absolute values between the reference method and the tested methods for each hemodynamic intervention.

\subsection{Trending ability}

The trending ability for Capno-SvO $\mathrm{S}_{2}$ and fiberoptic $\mathrm{SvO}_{2}$, compared to $\mathrm{CO}$-oximetry, are presented as four-quadrant plot illustrations in Fig. 4.

For Capno-SvO 2 , a total of 45 data points were found to lie outside the predefined $10 \%$ exclusion zone. 44 out of 45 values changed in the same direction as the reference method CO-oximetry which generates a concordance rate of $98 \%$. The corresponding data for fiberoptic $\mathrm{SvO}_{2}$ was 46 data points outside the exclusion zone with 43 data points moving in the same direction as the reference method i.e., a concordance rate of $93 \%$.
Fig. 3 Bland-Altman plots for pooled recordings for Capno$\mathrm{SvO}_{2}$ vs. CO-oximetry (A) and fiberoptic $\mathrm{SvO}_{2}$ vs. CO-oximetry $(\mathbf{B})$. Dotted line represents bias, blue dotted lines represent upper and lower limits of agreement and black broken lines represents the corresponding CI for the limits of agreements. $\mathrm{N}=11$. A 271 paired data points, B 272 paired data points

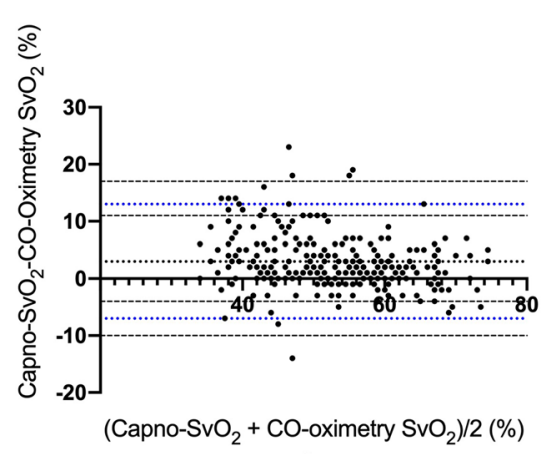

A

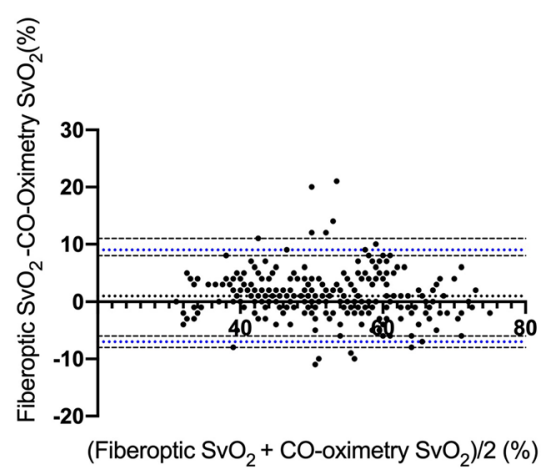

B

Table 1 Bias and limits of agreement between Capno- $\mathrm{SvO}_{2}$ and $\mathrm{CO}$-oximetry $\mathrm{SvO}_{2}$ as well as between fiberoptic and $\mathrm{CO}$-oximetry $\mathrm{SvO}$ for the various hemodynamic challenges

\begin{tabular}{|c|c|c|c|c|}
\hline Hemodynamic intervention & Compared methods & $\begin{array}{l}\text { Bias (percentage } \\
\text { points) }\end{array}$ & $\begin{array}{l}\text { ULOA }(\mathrm{CI}) \text { (percent- } \\
\text { age points) }\end{array}$ & LLOA $(\mathrm{CI})$ (percentage points) \\
\hline \multirow[t]{2}{*}{ Baseline precision } & Capno vs. CO-oxi & 2 & $10(7$ to 16$)$ & $-6(-12$ to -3$)$ \\
\hline & Fiber vs. CO-oxi & 2 & $10(7$ to 15$)$ & $-6(-12$ to -4$)$ \\
\hline \multirow[t]{2}{*}{$\mathrm{FiO}_{2}$} & Capno vs. CO-oxi & 1 & $11(8$ to 18$)$ & $-8(-15$ to -5$)$ \\
\hline & Fiber vs. CO-oxi & 0 & $7(7$ to 11$)$ & $-7(-12$ to -5$)$ \\
\hline \multirow[t]{2}{*}{ Hemorrhage } & Capno vs. CO-oxi & 3 & 14 (10 to 23$)$ & $-8(-17$ to -4$)$ \\
\hline & Fiber vs. CO-oxi & 2 & 13 (9 to 20 ) & $-8(-15$ to -4$)$ \\
\hline \multirow[t]{2}{*}{ Crystalloid infusion } & Capno vs. CO-oxi & 6 & $17(14$ to 23$)$ & $-6(-12$ to -3$)$ \\
\hline & Fiber vs. CO-oxi & 3 & $10(8$ to 15$)$ & $-3(-8$ to -1$)$ \\
\hline \multirow[t]{2}{*}{ Transfusion } & Capno vs. CO-oxi & 1 & $7(5$ to 11$)$ & $-5(-8$ to -3$)$ \\
\hline & Fiber vs. CO-oxi & 1 & $9(7$ to 14$)$ & $-8(-13$ to -6$)$ \\
\hline \multirow[t]{2}{*}{ Cava balloon } & Capno vs. CO-oxi & 5 & 13 (10 to 19$)$ & $-4(-10$ to -1$)$ \\
\hline & Fiber vs. CO-oxi & 1 & 6 (4 to 9$)$ & $-6(-8$ to -3$)$ \\
\hline \multirow[t]{2}{*}{ Dobutamine infusion } & Capno vs. CO-oxi & 5 & 11 (8 to 17$)$ & $-2(-7$ to 1$)$ \\
\hline & Fiber vs. CO-oxi & -1 & 8 (4 to 16$)$ & $-10(-18$ to -6$)$ \\
\hline
\end{tabular}

ULOA upper limits of agreement, LLOA lower limits of agreement, $\mathrm{CI} 95 \%$ confidence interval, Capno $\mathrm{Capno}_{\mathrm{SvO}}$, $\mathrm{CO}$-oxi $\mathrm{CO}$-oximetry $\mathrm{SvO}_{2}$, Fiber fiberoptic $\mathrm{SvO}_{2}$ 
Fig. 4 Four quadrant plots presenting the concordance between Capno- $\mathrm{SvO}_{2}$ and CO-oximetry $\mathrm{SvO}_{2}(\mathbf{A})$ and between fiberoptic $\mathrm{SvO}_{2}$ and CO-oximetry $\mathrm{SvO}_{2}(\mathbf{B})$. A 44 paired delta values, B 46 paired delta values. The central boxed area illustrates the $10 \%$ exclusion zone. Dotted line is line of identity. $\mathrm{N}=11$

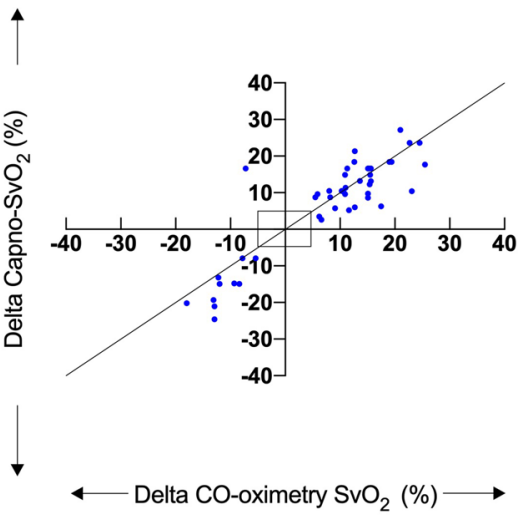

A

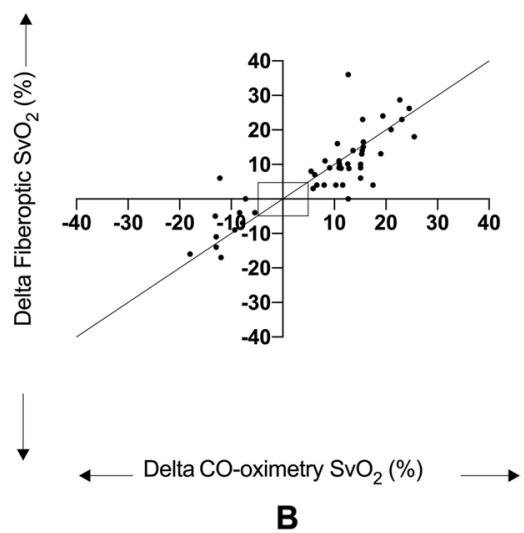

B

\section{Discussion}

The main finding of the current study was that Capno-SvO generates absolute values close to the gold standard $\mathrm{CO}$ oximetry during major hemodynamic provocations. The agreement between Capno-SvO $\mathrm{S}_{2}$ and CO-oximetry was comparable to that found for fiberoptic $\mathrm{SvO}_{2}$ vs. CO-oximetry. Furthermore, Capno- $\mathrm{SvO}_{2}$ demonstrated a slightly more accurate ability to detect change in $\mathrm{SvO}_{2}$ than fiberoptic $\mathrm{SvO}_{2}$.

\subsection{Agreement of absolute values between CO-oximetry and the tested methods}

Reliability in consistency during repeated measures and the ability to generate absolute values that corresponds acceptably with the gold standard can be considered absolute prerequisites for any new monitoring technique. During stable baseline conditions, Capno-SvO $\mathrm{S}_{2}$ displayed an inherent precision matching those of both CO-oximetry and fiberoptic $\mathrm{SvO}_{2}$, thus, indicating a reliable monitoring system. During the following hemodynamic interventions Capno- $\mathrm{SvO}_{2}$ generated values close to the gold standard method and the overall agreement between Capno- $\mathrm{SvO}_{2}$ and $\mathrm{CO}$-oximetry was within the predefined $95 \%$ limits of agreement of $15 \%$ points and well below the a priori determined limit for a $\mathrm{PE}$ of $<30 \%$. There was an overall tendency for Capno$\mathrm{SvO}_{2}$ to display slightly higher $\mathrm{SvO}_{2}$ values compared to $\mathrm{CO}-$ oximetry even if this difference was relatively small and within clinically acceptable limits. Fiberoptic $\mathrm{SvO}_{2}$ showed a smaller bias and narrower limits of agreement against $\mathrm{CO}$-oximetry than that recorded for Capno- $\mathrm{SvO}_{2}$. Even if the difference in agreement against gold standard was relatively similar for both methods, the fact that fiberoptic $\mathrm{SvO}_{2}$ showed slightly better agreement is not entirely surprising since this value is generated from direct estimation of $\mathrm{SvO}_{2}$ in the actual pulmonary artery whereas the Capno- $\mathrm{SvO}_{2}$ is a figure derived from mathematical analysis of an induced variability exhaled $\mathrm{CO}_{2}$.

Both Capno- $\mathrm{SvO}_{2}$ and fiberoptic $\mathrm{SvO}_{2}$ showed variable levels of agreement against CO-oximetry depending on the type of hemodynamic intervention. During stable baseline conditions, both Capno- $\mathrm{SvO}_{2}$ and fiberoptic $\mathrm{SvO}_{2}$ showed near enough equal agreement against gold standard. This pattern was repeated for the $\mathrm{FiO}_{2}$ step which is reassuring since increased $\mathrm{FiO}_{2}$ is a common method of augmenting oxygen delivery in the clinical setting.

Differences between Capno-SvO $\mathrm{S}_{2}$ and CO-oximetry can be attributed to mainly two factors. Firstly, issues related to $\mathrm{CO}_{\mathrm{EPBF}}$ calculations i.e., disturbances in the prerequisites for the capnodynamic method, mainly the assumption of stable or slowly varying mixed venous $\mathrm{CO}_{2}$ content and the use of updated $\mathrm{Hb}$ levels. Secondly the incorporated RQ value will affect the Capno- $\mathrm{SvO}_{2}$ and thus its level of agreement against CO-oximetry.

The relatively high bias and limits of agreements seen during the fluid resuscitation (especially data points 0 and $5 \mathrm{~min}$ ) and cava occlusion step is most likely due to initial disturbances in the mixed venous $\mathrm{CO}_{2}$ content and $\mathrm{Hb}$ level.

\subsection{Ability to track changes in $\mathrm{SvO}_{2}$ for Capno-SvO and fiberoptic $\mathrm{SvO}_{2}$}

Agreement of absolute values is central, but in the clinical context trending ability may be even more important [25]. In this study, Capno-SvO $\mathrm{S}_{2}$ showed a very good concordance rate when compared to the gold standard, detecting $98 \%$ of the changes, which is well in line with the established fiberoptic method. In addition, the agreement between $\mathrm{CO}$ oximetry and Capno- $\mathrm{SvO}_{2}$ during stable baseline conditions was very good. Appropriate agreement during stable conditions in combination with a high concordance rate against $\mathrm{CO}$-oximetry, makes Capno-SvO $\mathrm{S}_{2}$ potentially interesting as an early warning sign of not only more major hemodynamic 
deteriorations but also minor hemodynamic changes (as shown in our previous publication) [8].

\subsection{Limitations}

Even if the Capno-SvO ${ }_{2}$ technique showed potentially promising performance in the current study, like most monitoring methods it has inherent limitations that needs to be considered and further investigated, particularly if applied in a clinical setting.

\subsubsection{Issues related to calibration of the fiberoptic method}

The fiberoptic $\mathrm{SvO}_{2}$ method was recalibrated against COoximetry four times during the protocol to optimize its performance as shown in Fig. 1. Normally, the fiberoptic system is intended to be calibrated once daily and not frequently recalibrated [26]. As a consequence of these frequent recalibrations, not corresponding to normal clinical routine, the subsequent fiberoptic $\mathrm{SvO}_{2}$ values will have affected the performance of the fiberoptic catheter in a positive way. The Capno- $\mathrm{SvO}_{2}$ was recalibrated at the same time points as the fiberoptic system but only against the current $\mathrm{Hb}$ level since the Capno- $\mathrm{SvO}_{2}$ method is independent from repeated alignment against gold standard CO-oximetry obtained mixed venous saturations (as is the case for fiberoptic $\mathrm{SvO}_{2}$ ).

\subsubsection{Influence of respiratory quotient}

The RQ value is an important determinant in estimating Capno-SvO $\mathrm{O}_{2}$ and will affect the agreement between Capno$\mathrm{SvO}_{2}$ and CO-oximetry. Mathematically, choosing a higher $\mathrm{RQ}$ will elevate the Capno-SvO $\mathrm{O}_{2}$ and vice versa. Statistically this affects accuracy, but not precision - i.e., bias will shift and will subsequently move upper and lower limit of agreements equally in parallel [14]. It will, however, not have an impact on the trending ability, maybe the most important feature of a monitoring method, as discussed above.

In this study the RQ was directly measured, and the mean value of all measurements was used for calculations of Capno- $\mathrm{SvO}_{2}$. In the clinical setting it is more likely that RQ will be taken from tabulated values since a direct measurement is impractical with currently available methods. Furthermore, RQ depends on fasting time and the nutritional substrate given and this needs to be taken into consideration when evaluating the recorded RQ [27, 28]. In the current study, the animals were fasted and kept on a low-level glucose maintenance infusion, a common practice during anaesthesia. The scenario may have been different if nutritional status and/or infusions had been altered regarding glucose concentration or type of substrate, something that can be of importance in the intensive care setting [27]. Despite this, using the mean RQ for the whole group still resulted in acceptable agreement and trending ability.

\subsection{Clinical applicability}

In its current form, the Capno-SvO $\mathrm{S}_{2}$ method requires controlled mechanical ventilation [7, 29]. However, most of the patients that would benefit from continuous non-invasive assessment of $\mathrm{SvO}_{2}$ will already be subjected to controlled mechanical ventilation both in the operating room and in the intensive care unit. Still, the fact that the method requires controlled ventilation will exclude patients that are spontaneously breathing or are treated with various modes of patient triggered ventilation.

Although Capno-SvO $\mathrm{S}_{2}$ has the potential to considerably expand the ability to continuously monitor $\mathrm{SvO}_{2}$ compared to today, it is important to emphasize that Capno- $\mathrm{SvO}_{2}$ is not a substitute for the proper use of a pulmonary artery catheter since it does not provide information regarding relevant pressure and resistance parameters. However, it could potentially provide real time $\mathrm{SvO}_{2}$ monitoring in situations where a pulmonary artery catheter is neither practical or justifiable due to its invasive nature, associated risks, and cost. Thus, the potential role of Capno- $\mathrm{SvO}_{2}$ deserves further translational studies to define its place in monitoring of patients that are cared for in the operating room or in intensive care.

In conclusion, the current study shows that the Capno$\mathrm{SvO}_{2}$ method is in close agreement with the gold standard CO-oximetry and displays good trending ability even during major changes in oxygen delivery. These features of the Capno- $\mathrm{SvO}_{2}$ method, in combination with its noninvasive nature, may offer a novel real time option for $\mathrm{SvO}_{2}$-monitoring. Even if a pulmonary artery catheter is of seminal importance for complete hemodynamic assessment, the Capno-SvO $\mathrm{S}_{2}$ method could facilitate more advanced hemodynamic surveillance when a pulmonary artery catheter is not warranted, technically possible (such as in pediatric practice) or even contraindicated, thereby acting as a useful adjunct to more traditional hemodynamic monitoring.

Supplementary Information The online version contains supplementary material available at https://doi.org/10.1007/s10877-021-00762-5.

Author contributions AS, JK, MK and P-AL: Study design, data collection, analysis and manuscript writing. MH and MW: study design, data collection and analysis and critical manuscript review.

Funding Open access funding provided by Karolinska Institute. This work was supported by the Karolinska Institute and partially by unrestricted grants from Maquet Critical Care $\mathrm{AB}$ and the regional agreement on medical training and research (ALF) between Stockholm County Council and the Karolinska Institute.

Data availability Not applicable. 
Code availability Not applicable.

\section{Declarations}

Conflict of interest Per-Arne Lönnqvist received an unrestricted research grant from Maquet Critical Care AB. Mats Wallin and Mark Konrad was employed by Maquet Critical Care AB at the time of the study. Magnus Hallbäck is employed at Maquet Critical Care AB. Anders Svedmyr and Jacob Karlsson report no conflicts of interest.

Ethical approval Authorization was granted from Uppsala Animal Ethics Committee (Uppsala, Sweden case number C75/16, chairperson Erik Göransson on August 26, 2016). The animals were handled in accordance with the animal experimentation guidelines of the Uppsala Animal Ethics Committee and Animal Research: Reporting of In Vivo Experiments (ARRIVE) guidelines.

Consent to participate: Not applicable.

Consent for publication: Not applicable.

Open Access This article is licensed under a Creative Commons Attribution 4.0 International License, which permits use, sharing, adaptation, distribution and reproduction in any medium or format, as long as you give appropriate credit to the original author(s) and the source, provide a link to the Creative Commons licence, and indicate if changes were made. The images or other third party material in this article are included in the article's Creative Commons licence, unless indicated otherwise in a credit line to the material. If material is not included in the article's Creative Commons licence and your intended use is not permitted by statutory regulation or exceeds the permitted use, you will need to obtain permission directly from the copyright holder. To view a copy of this licence, visit http://creativecommons.org/licenses/by/4.0/.

\section{References}

1. Holm J, Håkanson E, Vánky F, Svedjeholm R. Mixed venous oxygen saturation predicts short- and long-term outcome after coronary artery bypass grafting surgery: a retrospective cohort analysis. $\mathrm{Br} \mathbf{J}$ Anaesth. 2011;107:344-50.

2. Kalra A, Heitner $\mathrm{S}$, Topalian S. Iatrogenic pulmonary artery rupture during Swan-Ganz catheter placement: a novel therapeutic approach. Catheter Cardiovasc Interv. 2013;81:57-9.

3. Hadian M, Pinsky MR. Evidence-based review of the use of the pulmonary artery catheter: impact data and complications. Crit Care. 2006;10(Suppl 3):8.

4. Karlsson J, Wallin M, Hallbäck M, Lönnqvist PA. Capnodynamic determination of cardiac output in hypoxia-induced pulmonary hypertension in pigs. Br J Anaesth. 2019;122:335-41.

5. Hällsjö Sander C, Hallbäck M, Wallin M, Emtell P, Oldner A, Björne $\mathrm{H}$. Novel continuous capnodynamic method for cardiac output assessment during mechanical ventilation. $\mathrm{Br} \mathbf{J}$ Anaesth. 2014;112:824-31.

6. Sigmundsson TS, Öhman T, Hallbäck M, Redondo E, Sipmann FS, Wallin M, Oldner A, Hällsjö Sander C, Björne H. Performance of a capnodynamic method estimating effective pulmonary blood flow during transient and sustained hypercapnia. J Clin Monit Comput. 2018;32:311-9.

7. Peyton PJ, Wallin M, Hallbäck M. New generation continuous cardiac output monitoring from carbon dioxide elimination. BMC Anesthesiol. 2019;19:28.

8. Karlsson J, Lönnqvist PA, Wallin M, Hallbäck M. A continuous noninvasive method to assess mixed venous oxygen saturation: a proof-of-concept study in pigs. Anesth Analg. 2021;132(6):176876. https://doi.org/10.1213/ANE.0000000000005188.

9. Karlsson J, Winberg P, Scarr B, Lönnqvist PA, Neovius E, Wallin M, Hallbäck M. Validation of capnodynamic determination of cardiac output by measuring effective pulmonary blood flow: a study in anaesthetised children and piglets. Br J Anaesth. 2018;121:550-8.

10. Ferrando C, Romero C, Tusman G, Suarez-Sipmann F, Canet J, Dosdá R, Valls P, Villena A, Serralta F, Jurado A, Carrizo J, Navarro J, Parrilla C, Romero JE, Pozo N, Soro M, Villar J, Belda FJ. The accuracy of postoperative, non-invasive Air-Test to diagnose atelectasis in healthy patients after surgery: a prospective, diagnostic pilot study. BMJ Open. 2017;7:e015560.

11. Suarez-Sipmann F, Bohm SH, Tusman G. Volumetric capnography: the time has come. Curr Opin Crit Care. 2014;20:333-9.

12. Fick A. Uber die messung des Blutquantums in den Hertzvent rikeln. Sitzber Physik Med Ges Wurzburg, 1870, pp 290-91.

13. Gedeon A, Krill P, Osterlund B. Pulmonary blood flow (cardiac output) and the effective lung volume determined from a short breath hold using the differential Fick method. J Clin Monit Comput. 2002;17:313-21.

14. Cecconi M, Rhodes A, Poloniecki J, Della Rocca G, Grounds RM. Bench-to-bedside review: the importance of the precision of the reference technique in method comparison studies-with specific reference to the measurement of cardiac output. Crit Care. 2009;13:201.

15. Bland JM, Altman DG. Statistical methods for assessing agreement between two methods of clinical measurement. Lancet. 1986;1:307-10.

16. Bland JM, Altman DG. Agreement between methods of measurement with multiple observations per individual. J Biopharm Stat. 2007; 17:571-82.

17. Critchley LA, Critchley JA. A meta-analysis of studies using bias and precision statistics to compare cardiac output measurement techniques. J Clin Monit Comput. 1999;15:85-91.

18. Herner A, Haller B, Mayr U, Rasch S, Offman L, Schmid R, Huber W. Accuracy and precision of $\mathrm{ScvO} 2$ measured with the CeVOXdevice: A prospective study in patients with a wide variation of ScvO2-values. PLoS One. 2018;13:e192073.

19. Kissoon N, Spenceley N, Krahn G, Milner R. Continuous central venous oxygen saturation monitoring under varying physiological conditions in an animal model. Anaesth Intensive Care. 2010;38:883-9.

20. Iodice FG, Ricci Z, Haiberger R, Favia I, Cogo P. Fiberoptic monitoring of central venous oxygen saturation (PediaSat) in small children undergoing cardiac surgery: continuous is not continuous. F1000Res. 2014;3:23.

21. Lu MJ, Zhong WH, Liu YX, Miao HZ, Li YC, Ji MH. Sample size for assessing agreement between two methods of measurement by Bland-Altman method. Int J Biostat. 2016;10:20. https://doi.org/10. 1515/ijb-2015-0039.

22. Critchley LA, Lee A, Ho AM. A critical review of the ability of continuous cardiac output monitors to measure trends in cardiac output. Anesth Analg. 2010;111:1180-92.

23. Porath M, Sinha P, Dudenhausen JW, Luttkus AK. Systematic instrumental errors between oxygen saturation analysers in fetal blood during deep hypoxemia. Clin Chim Acta. 2001;307:151-7.

24. Beards SC, Edwards JD, Nightingale P. The need for quality control in measurement of mixed venous oxygen saturation. Anaesthesia. 1994;49:886-8.

25. Shepherd SJ, Pearse RM. Role of central and mixed venous oxygen saturation measurement in perioperative care. Anesthesiology. 2009;111:649-56.

26. LLC EL. Edwards hemosphere advanced monitor operator's manual 2017. https://www.iccueducation.org.uk/uploads/2/3/1/0/23109338/ hemosphere_cardiac_output_monitor.pdf. Accessed 25 Mar 2021 
27. Frankenfield DC. Impact of feeding on resting metabolic rate and gas exchange in critically ill patients. J Parenter Enteral Nutr. 2019;43:226-33.

28. Liu H, Chen Y, Ming D, Wang J, Li Z, Ma X, van Milgen J, Wang F. Integrative analysis of indirect calorimetry and metabolomics profiling reveals alterations in energy metabolism between fed and fasted pigs. J Anim Sci Biotechnol. 2018;9:41.

29. Sander CH, Sigmundsson T, Hallbäck M, Sipmann FS, Wallin M, Oldner A, Björne H. A modified breathing pattern improves the performance of a continuous capnodynamic method for estimation of effective pulmonary blood flow. J Clin Monit Comput. 2017;31:717-25.

Publisher's Note Springer Nature remains neutral with regard to jurisdictional claims in published maps and institutional affiliations. 